\title{
Combustion Noise Reduction using Multiple Injection Strategy in Diesel Engine
}

\author{
Shriniwas D. Chivate* and Y. P. Reddy \\ †Mechanical Engineering, Savitribai Phule Pune University, Pune, India \\ Accepted 15 June 2016, Available online 20 June 2016, Special Issue-5 (June 2016)
}

\begin{abstract}
The invention of common rail fuel injection system in diesel engine has become a prominent solution to many emission problems owing to provision of liberty in multiple injections. The complex combustion mechanism in diesel engines produces combustion noise which adds up to mechanical noise to give total radiated noise. The presented work involves the assessment of effect of injection strategy modification on combustion noise. The combustion noise of baseline injection strategy is evaluated using a software tool. The injection parameters such as pilot injection fuel quantity and separation, main injection timing, rail pressure are modified to arrive at minimum noise producing combination at various speed and load conditions. The combustion noise is evaluated again with modified injection strategy. The combustion noise is found to be reduced by maximum of $4 \mathrm{~dB}$. The total noise is also reduced by $1 \mathrm{~dB}$ at lower speed and loads. Structural attenuation is found to be same as expected. This approach will provide an alternative to time consuming and costly approach of structural modification to reduce the radiated noise. As a novel activity, a neural network and Graphical User Interface is developed for prediction of radiated noise and sound quality parameters when cylinder pressure is provided as an input. The trained neural network is found to give good co-relation between target values and predicted values. This network can be effectively used for noise and sound quality prediction using only cylinder pressure and speed as an input.
\end{abstract}

Keywords: Combustion noise, common rail diesel engine, function fitting, feed forward neural network, injection strategy, structural attenuation.

\section{Introduction}

The Electronic Fuel Injection system has become a prominent solution to cater the needs of engine developers to lower the emission level and combustion noise. Bosch introduced first Common Rail Direct injection (CRDi) which allowed multiple injections in diesel engines. Common Rail (CR) system has made it possible to use more than one injections independent of engine speed. The injection parameters such as pilot injection timing and fuel quantity, main injection timing, injection pressure can be altered at will in CR systems (Ulrich Flaig et al, 1999), (Kumbhar M.S. et al, 2010). This injection strategy has proved to be useful in reducing emission levels but at the same time these injections influences the in-cylinder pressure and consequently radiated noise. Fuel combustion produces the so-called combustion noise. The unpleasant sound signature of diesel engines is due to the harsh and irregular self ignition of the fuel. Therefore, being able to extract combustion noise from overall noise is of prime interest. The residual noise is referred to as mechanical noise. Depending upon

*Corresponding author: Shriniwas D. Chivate contribution of these noises to overall noise, a methodology is adopted to reduce the noise level of the engine. Several methods have been proposed to separate these noises (Chris Carlson et al, 2011) (Randolf A. et al, 2009),(Izuho Hirano et al, 1999). No work is reported on quantification of combustion noise reduction. The current study uses the multiple regression analysis to calculate the contribution of combustion and mechanical noise to the total radiated noise. The noise contribution at existing injection strategy is evaluated and injection strategy modification activity is exercised. The noise contribution evaluation is repeated with modified injection strategy and results are analyzed.

The NVH characteristics of the vehicle and engine are no more a secondary purchasing criteria's these days. Customer requirement of comfort has forced the designers to consider the NVH aspects of powertrain in the design stage itself. The function fitting property of neural networks is being used in many applications (Shumming Chen et al, 2013). Prediction of powertrain radiated noise in design stage itself is yet to achieved with accuracy. In a novel idea to implement, a neural network is developed which when trained with 
sufficient data points, can predict the radiated noise and 5 sound quality parameters when cylinder pressure and speed are used as inputs.

\section{Theoretical Background}

The radiated diesel powertrain noise consist of combustion and mechanical noise as primary sources as the intake and exhaust noise are suppressed during radiated noise measurement.

\subsection{Combustion Noise}

The explosive combustion of diesel fuel in the combustion chamber creates the irritating noise perception. The combustion noise is always governed by the property called Structural attenuation(SA) of the engine structure. The combustion noise is simply the subtracting the structural attenuation spectrum of the engine from in-cylinder pressure spectrum. This implies that higher the structural attenuation the lower will be the combustion noise. Apart from SA, the combustion noise is controlled by cylinder pressure which in turn depend upon the injection parameters like main injection timing, main injection fuel quantity, pilot injection fuel quantity and separation, rail pressure etc. The total noise reduction is only possible if the contribution of combustion noise to total noise is significant.

\subsection{Mechanical Noise}

The mechanical noise comprise of any noise due to impact of engine components during operation, resonances of various components, noise radiated from relative motion between the two components etc. The noise radiated from timing belt system, lubrication system, cooling system, valve timing system, piston slap phenomenon, bearing system, auxiliary systems like power steering pump, AC compressor is categorised as mechanical noise. The mechanical noise can only be controlled either by structural modification of resonating components or by proper natural frequency tunning of engine components. Out of several methods being used for combustion and mechanical noise separation, multiple regression analysis is used in this work.

\subsection{Noise Separation using Multiple Regression}

The approach for assessing noise contributions is based on following mathematical formulation. Assuming that engine noise consists of mechanical noise, combustion noise and load-dependent noise, it can be expressed as

$\mathrm{SP}=\mathrm{SP}_{\mathrm{m}}+\mathrm{H} \times \mathrm{CP}+\mathrm{G} \times \mathrm{L}$

where SP is the engine noise(power), SPm is the mechanical noise(power), $\mathrm{CP}$ is the cylinder pressure(power), $\mathrm{H}$ is the transfer coefficient between the cylinder pressure (power) and the combustion noise (power), $\mathrm{L}$ is the square of engine torque and $\mathrm{G}$ is the transfer coefficient between torque and loaddependent noise. In multivariate analysis terminology, SP is a criterion variable, CP and L are explanatory variables and SPm, $\mathrm{H}$ and $\mathrm{G}$ are partial regression coefficients. Consider that there are $\mathrm{n}$ sets of SP, CP and $\mathrm{L}$ data $\left(\mathrm{SP}_{1}, \mathrm{CP}_{1}, \mathrm{~L}_{1}\right)-\left(\mathrm{SP}_{\mathrm{n}}, \mathrm{CP}_{\mathrm{n}}, \mathrm{L}_{\mathrm{n}}\right)$ and arbitrary values of $\mathrm{SP}_{\mathrm{m}}, \mathrm{H}$ and $\mathrm{G}$. When these are substituted into above equation, it is assumed that they have error of $\mathrm{e}_{1}, \mathrm{e}_{2}, \ldots$, $e_{n}$ as indicated in the following equation. These terms can be expressed in matrix form as shown below.

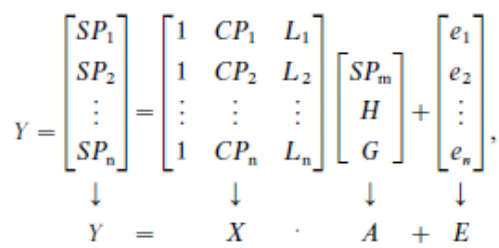

where $\mathrm{Y}$ is a noise vector, $\mathrm{X}$ is an excitation force matrix, A is a coefficient vector that also includes mechanical noise and $\mathrm{E}$ is an error vector. The object of a multiple regression analysis is to minimize the square ETE of the error vector in above matrix form. In other words, the value of vector $A$ is determined by the least-squares method and can be found in this case as

$$
A=\left(X^{T} \times X\right)-1 \times X^{T} \times Y
$$

By multiplying the excitation force matrix $\mathrm{X}$ by the transfer coefficient vector $A$ thus found, the noise vector $\hat{Y}$ is obtained as a calculated value, making it possible to separate mechanical noise, combustion noise and load dependent noise as follows:

$$
\hat{\mathrm{Y}}=\mathrm{X} \times \mathrm{A}
$$

The frequency characteristics of mechanical noise, combustion noise and load-dependent noise are then found by performing this calculation for every center frequency. The cylinder pressure data and noise measurement data is also used by some researchers to perform at combustion noise analysis.

\section{Experimentation}

The experimental setup used for testing is shown is Fig 1. Experimental setup consist of a Hemi-anechoic chamber which is designed to simulate free field condition for noise measurement.

A high speed common rail diesel engine is mounted similar to that of vehicle level. Intake and exhaust are taken out of the chamber using hoses so that only bare engine radiated noise is measured. The engine is connected to the dynamometer situated outside the chamber. 4 microphones on left, right front and top 
side of the engine are mounted at $1 \mathrm{~m}$ distance as shown in figure 1 . The testing for this study started with radiated noise measurement to estimate the combustion noise contribution. This is accomplished with following methodology-

The engine is tested at constant speed with load on the engine at that speed is varied from 0 to $100 \%$. This process is repeated at each suitable speed step. The cylinder pressure and engine torque is also measured. The acquired test data is processed to arrive at contribution of combustion and mechanical noise to overall noise. This gives the contributions for baseline injection strategy.

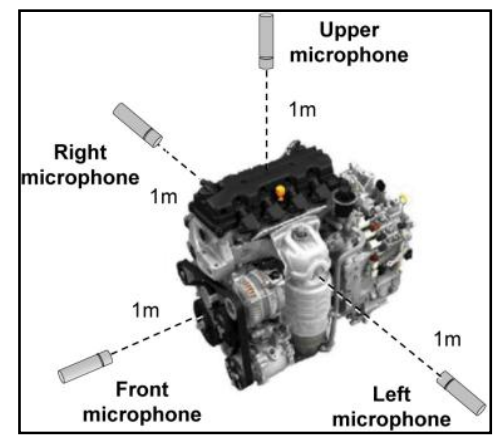

Fig. 1 Experimental setup for noise measurement

Once the contributions are known, the potential speed and load conditions can be identified at which the combustion noise is dominant and has a prospective for combustion process amendment. There is a potential for noise reduction at all speed and load conditions and injection parameter change is exercised for all conditions. Once the injection parameters are chosen to give the least noise for all speed and load conditions, the combustion and mechanical noise separation is again performed now with the modified injection strategy but with some smoothing in parameters. The baseline and new noise characteristics are compared.

\section{Result Discussion}

$1 / 3$ octave spectrum of radiated noise and in-cylinder pressure for old and modified injection strategy at a particular speed and load condition is shown in figure 2 and 3.



Fig. 2 Effect of injection parameter modification on SPL
It is clear from Fig. 2 and Fig. 3 that injection strategy influences the mid and high frequency spectrum of cylinder pressure but in SPL spectrum this change is reflected on in mid frequency range the reason being very high level of structural attenuation in low and high frequency range.

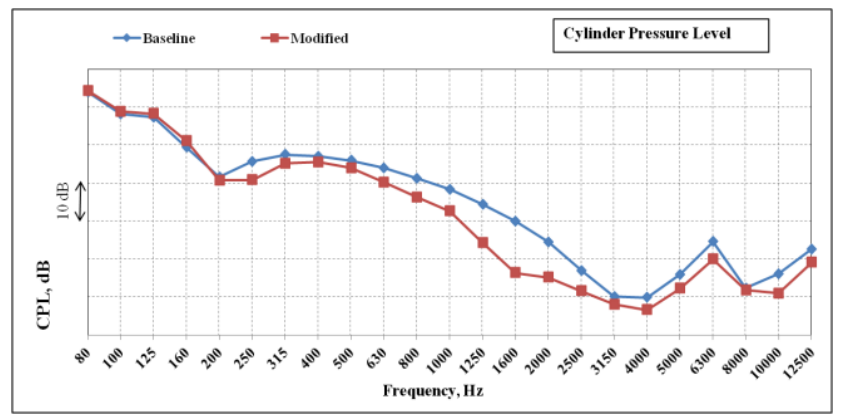

Fig.3 Effect of injection parameter modification on CPL

The combustion and mechanical noise separation analysis is exercised twice, one with the old injection parameters and one after the injection parameters are changed to reduce the noise. The noise reduction activity carried out with injection parameters modification is implemented at each speed and load condition and the combination giving the minimum noise are finalized. When this activity is finished, once again the combustion and mechanical noise separation is evaluated but now with the new injection parameters. The comparative analysis of old and new combustion and mechanical analysis is found to be inline with the results of steady noise measurement wherein the modified combinations of injection parameters reduced the radiated noise. There is a decrease in the radiated noise level with new injection parameters. Almost all load and speed conditions have marked a reduction in the radiated noise. The peculiar observation is combustion noise is affected more than mechanical noise.

The comparative study and analysis of baseline and modified injection strategy is described as follows.

\subsection{Total noise}

The rate of pressure rise is the key factor amongst all combustion parameters which affect the radiated noise the most. Higher the rate of pressure rise higher the radiated noise. The effect of injection parameter modification at all load conditions except $0 \%$ load is shown in Fig. 4. The modification of injection parameters has found to effective in low load conditions.

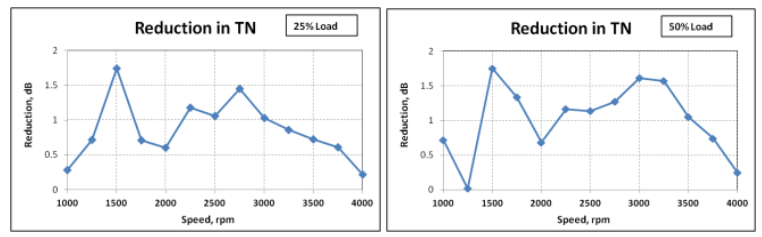




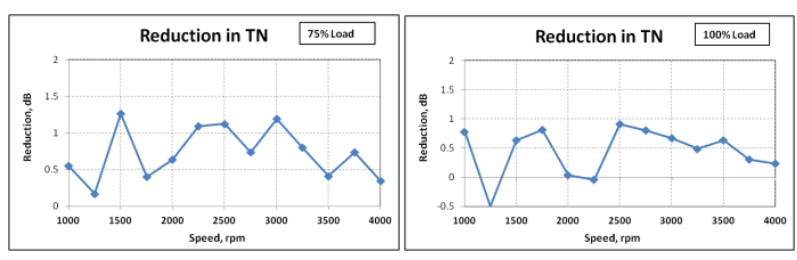

Fig. 4 Effect of injection strategy on total noise

The highest noise reduction of 1-1.7 $\mathrm{dB}$ is achieved at $50 \%$ load which is evident from the graph. However at higher loads, only marginal effect of 0-0.5 dB reduction is observed. Furthermore at very low(1000 and 1250 rpm) and very high speeds(3750 and 4000rpm) the effect is not significant. The reason of this ineffectiveness can be attributed to small reduction in combustion noise at that speed. Though the reduction in SPL is less the audio perception of radiated noise of the baseline and modified injection strategy shows the significant improvement.

\subsection{Combustion noise}

As the motive behind the injection parameter modification is to influence combustion process and consequently combustion noise, it was obvious to that combustion noise will get affected the most. The comparison of combustion noise with old and modified injection parameters establish the assumption. The Fig. 5 confirm that the combustion noise has reduced by 0.5 - $5 \mathrm{~dB}$ at certain speed and load conditions. At very low(1000 and $1250 \mathrm{rpm}$ ) the effect is not significant as in the case of total radiated noise. The trend of noise reduction at the low load conditions is similar to that in total noise i.e. highest decrease in combustion noise is observed at $25 \%$ and $50 \%$ load conditions. The injection parameter modification has found to be more beneficial in the speed range of $1500-3500 \mathrm{rpm}$. In some speed and load conditions the $\mathrm{CN}$ increased.

\subsection{Mechanical noise}

The effect of injection parameter modification on mechanical noise at different load conditions is also found out.

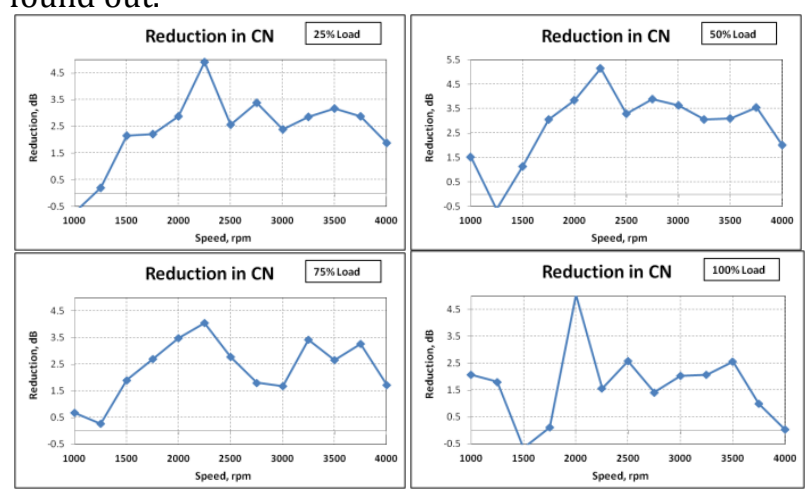

Fig. 5 Effect of injection strategy on combustion noise

It is evident from the results that mechanical noise is least affected by modification. Although the effect of modification is small, out of given load conditions, mechanical noise has shown maximum effect at 50\% load condition. The injection parameter modification has reduced mechanical noise by small amount 0.3 $1 \mathrm{~dB})$. At all other loads except $50 \%$ the effect of modification on mechanical noise is negligible. As all the operating conditions are same and only combustion process is altered, the mechanical noise should ideally remain same. The results are inline with that presumption. However whatever reduction in mechanical noise is observed is because forcing function i.e. combustion excitation is reduced.

\subsection{Structural Attenuation (SA)}

Structural Attenuation is a property of the engine structure by virtue of which cylinder pressure excitation will get attenuated. The Structural Attenuation(SA) is calculated using the software tool for both old and modified injection parameters.

SA being low, it's because of this property, the Sound Pressure Level(SPL) of the engine is high in the range of 1000-4000 Hz range. The cylinder pressure level in below $1000 \mathrm{~Hz}$ range is quite large but due to high SA, the radiated noise in this range is low. The cylinder pressure level in above $4000 \mathrm{~Hz}$ range is relatively less at the same time the SA is very high which is the reason of low noise radiation in above $4000 \mathrm{~Hz}$ frequency range.

The SA curves of old and modified condition matches with negligible deviation. Owing to the fact that same engine is used for measurement in both conditions, the SA curve should match and it has validated the measurement. SA is an important entity in combustion noise analysis as it governs the contribution of combustion and mechanical noises. It an essential property of the engine structure while distinguishing one engine structure from another. If the attenuation is high in a particular frequency band the combustion noise in that band will be less and vice versa. Depending upon the results and analysis of radiated noise and structural attenuation it can also be concluded that whether the injection parameter modification will reduce the noise or not.

\section{Application of Neural Network}

The artificial neural networks are gaining more and more popularity amongst the researchers due to its versatile capabilities in solving function fitting, pattern recognition, classification and clustering problems The presented research utilizes the function fitting property of neural networks. It is said by many researchers that even the simplest form of neural network can fit almost any input and output data. This has led to the tremendous increase in applications of neural networks in almost all fields. 
The neural network toolbox in MATLAB has a function fitting capability which can be invoked by using nftool command. If a black box concept is applied to an engine noise problem, the simplest model will have cylinder pressure, no. of cylinders and engine speed as an input and radiated noise and other entities such as SQ parameters, emission parameters etc. will be the output. For presented study, the frequency domain cylinder pressure data is utilized. The neural network model is developed in such a way that input to the model is $1 / 3$ octave band spectrum of cylinder pressure. As this neural network is set up for a particular engine, no. of cylinders in the engine can be ignored. However, there could be different engines for which similar network can be designed and in that case no. of cylinders will be an indispensable parameter. The output is radiated Sound Pressure Level(A weighted), Loudness Zwicker, Roughness, Sharpness, Articulation Index and Fluctuation Strength. The experimental measurements are carried out for above parameters at different load and speed conditions. The dataset is prepared for 'Inputs' and 'Targets' which is a pre-requisite for neural network processing. Neural network training procedure is completed.

A function is required to be fitted between incylinder pressure of a diesel engine at specific speed and load and 6 output parameters such as Radiated Sound Pressure Level(SPL), Loudness Zwicker, Sharpness, Articulation Index, Fluctuation Strength and Roughness. These parameters are experimentally measured in a hemi-anechoic chamber or processed offline using the noise measurement data. The cylinder pressure is presented in $1 / 3$ octave band in frequency domain. The reason being noise analysis is more informative if it is carried out in frequency domain. In the input dataset, apart from cylinder pressure, speed is also added as the radiated noise is strongly dependant on speed of the engine. Each input vector has 38 elements in it. The output vector consists of 6 elements, SPL and 5 Sound Quality matrices as mentioned above. The dataset contains 400 measurements which will be used for network training. A specialized two layer feedforward neural network known as fitnet is created in MATLAB using neural network toolbox. This fitnet network has been developed for function fitting. The hidden layer transfer function is set as hyperbolic tangent sigmoid transfer function tansig and linear transfer function purelin is used for output layer.

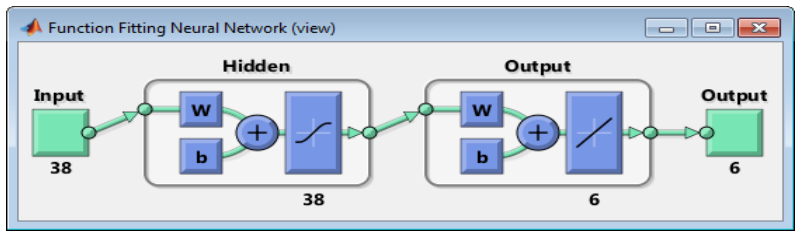

Fig. 6 Two layer feedforward neural network
The number of neurons in the hidden layer are kept at 38.The number of neurons can be changed but to begin with the training, one neuron for one input is decided. The numbers of neurons in output layers are always equal to number of output which is 6 in this case. The neural network used in this application is shown in Fig 6.

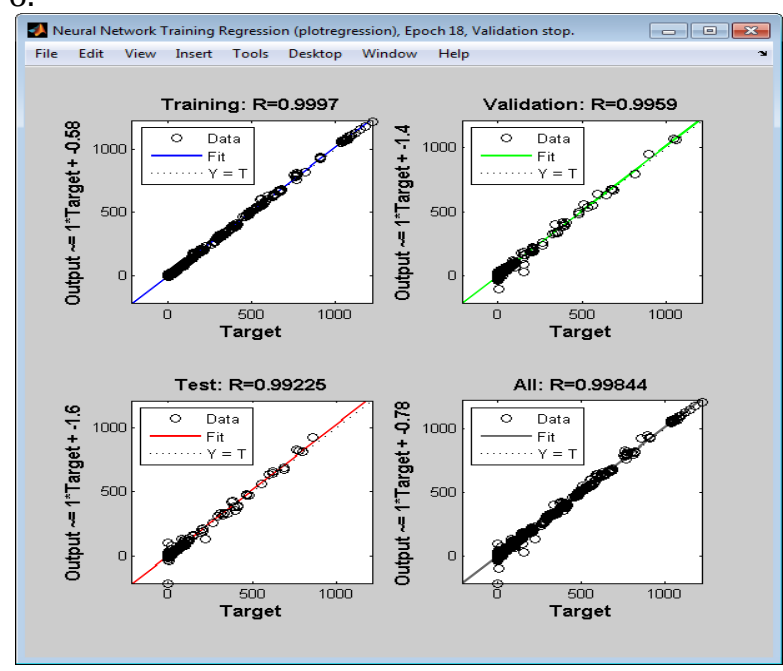

Fig. 7 Co-relation co-efficient

Out of total available samples $70 \%$ of the samples are utilized for training the network, $15 \%$ of the samples are used for validation and rest $15 \%$ are used for testing. The fastest Levenberg-Marquardt algorithm is selected for training the network. For error calculation and performance of network mean square normalized error function mse is chosen. After the training is completed, its training performance is checked. For training to be satisfactory, the co-efficient of corelation(R) between targets and output of the network should be close to 1 . The $R$ value is found to be very close to 1 for all three training, validation and testing categories of dataset.

However the error value after the training is found to be of concern. The SPL and loudness level values are bigger than rest 4 parameters but the error criteria is same for all output parameters. This may cause the major shift in output whose target values are very small. To rectify this problem, the training set is modified and the target parameters are multiplied by a factor so that its value becomes comparable to SPL and loudness. The output of new network should be divided by same factor before displaying these values to GUI. This activity of scaling the output has improved the error performance made the network ready for prediction of data for given input. The graphs of $\mathrm{R}$ for different dataset categories and overall regression plot are shown in figure 7.

The Graphical User Interface (GUI) is created in MATLAB to display the predicted output of neural network. A pushbutton is provided which on pressing, displays the output. The GUI is shown in figure 8. 


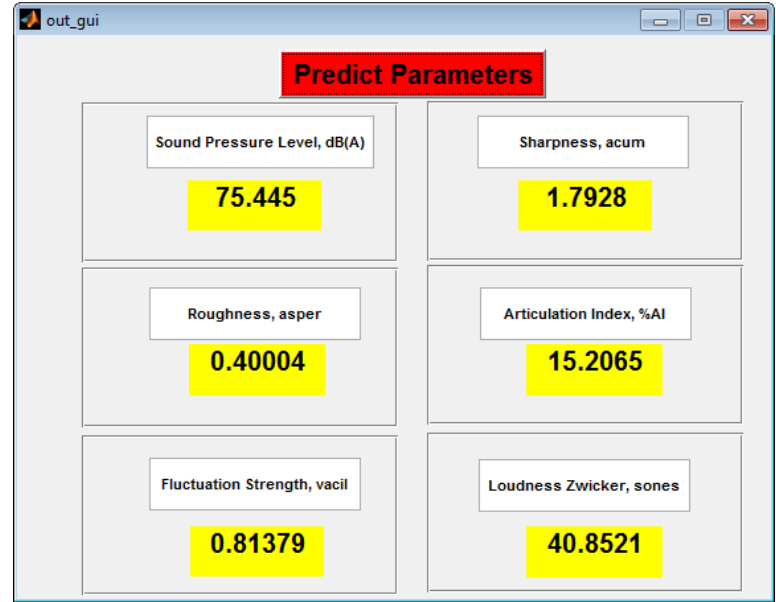

Fig. 8 GUI for output

\section{Conclusions}

The common rail injection system is found to be very effective in changing the injection parameters at will. After the change in injection parameters at all speed and all load conditions to reduce combustion noise, combustion mechanical noise separation revealed that both combustion noise and radiated noise are reduced. The structural attenuation (SA) is found to be same for both before and after analysis. The noise reduction strategy based on combustion noise reduction is very effective in common rail engines as it doesn't demand any structural modification which supposed to be very time consuming and costly affair.

However it should also be emphasized that the potential for noise reduction by altering injection strategy is comparatively less. This methodology has great influence on sound quality of the radiated noise.
The function fitting activity performed using neural network is an innovative approach which can actually reduce the experimentation required for further assessment of noise by changing injection strategy. Even only cylinder pressure and speed measurement can lead to radiated noise and sound quality parameter prediction once proper fit is achieved.

\section{References}

Ulrich Flaig, Wilhelm Polach and Gerhard Ziegler(1999), Common Rail System (CR-System) for Passenger Car DI Diesel Engines; Experiences with Applications for Series Production Projects, SAE International, 1999-01-0191

Kumbhar M. S., Rajkumar P. B., Velayudham N. and Kiran Mohan(2010),Powertrain Noise \& Sound Quality Refinement for New Generation Common Rail Engines, SAE International, 2010-01-1414

Izuho Hirano, Masahiko Kondo, Youichi Uraki, Yasuyuki Asahara(1999), Using multiple regression analysis to estimate the contributions of engine-radiated noise components, JSAE Review 20 (1999) 363-368

Randolf A., Hans-Herwig P., Franz B. and Jürgen Veit(2009), Procedure for Separating Noise Sources of Combustion Engines, SAE International, 2009-26-054

Chris A Carlson and Dhanaji J Kalsule(2010), Combustion Mechanical Breakdown: A Comparison of the Multiple Regression Method versus the Coherence Method for a HSDI Diesel Powertrain, SAE International, 2011-26-0035

Shuming Chen, Dengfeng Wang, Yinchong Wu, Zongwei Liu and Hailin Wang(2013), Objective evaluation of interior sound quality in passenger cars using artificial neural networks, SAE International journal of Passenger cars, 2013-01-1704 